\title{
Análise epidemiológica de casos de sífilis notificados em uma cidade do interior paulista
}

\author{
Epidemiological analysis of reported syphilis cases in a city in the interior of State of Sao \\ Paulo, Brazil
}

\section{Análisis epidemiológico de los casos de sífilis notificados en una ciudad del interior del Estado de São Paulo, Brasil}

Caio Roberto de Paiva Moreno ${ }^{1 *}$, Guilherme da Mata Freitas ${ }^{1}$, Maurício Manuel Llaguno Lazo.

\section{RESUMO}

Objetivo: Descrever o perfil clínico epidemiológico dos pacientes acometidos por sífilis (adquirida, congênita e gestacional), e suas nuances. Métodos: Trata-se de um estudo observacional descritivo retrospectivo, analisando os perfis epidemiológicos dos casos de sífilis notificados pela Vigilância Epidemiológica Municipal, utilizando-se do Sistema de Informação de Agravos de Notificação (SINAN), no período de 2009 a 2018, no município de Franca (SP). Resultados: A frequência dos casos dessa infecção sexualmente transmissível no município apresentou tendência crescente. Além disso, pode-se constatar mudanças decorrentes da notificação compulsória de sífilis adquirida a partir de 2010. Analisou-se também a grande necessidade e relevância do pré-natal, além da complexidade do tratamento completo adequado, abordando o tratamento do parceiro ou parceira. Tal estudo apresenta uma abordagem ampla e completa sobre o perfil epidemiológico de sífilis no munícipio situado no interior paulista, apesar da subnoficação e do preenchimento incompleto e/ou inadequado das fichas de notificação. Conclusão: Conclui-se que existe uma necessidade de melhoria nas ações para prevenção da sífilis nos serviços de saúde da atenção básica.

Palavra-chave: Sífilis, Epidemiologia, Infecção sexualmente transmissível.

\section{ABSTRACT}

Objective: To describe the clinical epidemiological profile of syphilis patients (acquired, congenital and gestational), and their nuances. Methods: This is a retrospective observational descriptive study, analyzing the epidemiological profiles of syphilis cases reported by the Municipal Epidemiological Surveillance, using the Acute Notification Information System (SINAN), from 2009 to 2018, in the municipality of Franca (State of Sao Paulo, Brazil). Results: The frequency of cases of this sexually transmitted infection in the municipality showed an increasing trend. In addition, changes can be seen from the compulsory notification from 2010 , of syphilis acquired. In addition, we analyzed the great need and relevance of prenatal care, in addition to the complexity of adequate complete treatment, addressing the treatment of the partner. This study presents a broad and complete approach on the epidemiological profile of syphilis in the municipality located in the interior of State of Sao Paulo, Brazil, despite the subnotification and incomplete and/or inadequate completion of notification forms. Conclusion: It is concluded that there is a need for improvement in syphilis prevention actions in basic care health services.

Keywords: Syphilis, Epidemiology, Sexually transmitted infection.

\section{RESUMEN}

Objetivo: Describir el perfil epidemiológico clínico de los pacientes con sífilis (adquirida, congénita y gestacional), y sus matices. Métodos: Se trata de un estudio descriptivo observacional retrospectivo, en el que se analizan los perfiles epidemiológicos de los casos de sífilis notificados por la Vigilancia Epidemiológica Municipal, utilizando el Sistema de Información de Notificaciones Agudas (SINAN), de 2009 a 2018, en el municipio de Franca, Estado de São Paulo, Brasil. Resultados: La frecuencia de los casos de esta infección de transmisión sexual en el municipio mostró una tendencia creciente. Además, se pueden observar cambios en la notificación obligatoria de la sífilis adquirida a partir de 2010. También analizamos la gran necesidad y relevancia de la atención prenatal, además de la complejidad de un tratamiento completo adecuado, que aborde el tratamiento de la pareja. Este estudio presenta un enfoque amplio y completo del perfil epidemiológico de la sífilis en el municipio situado en el interior del Estado de São Paulo, a pesar de la subnotificación y de la cumplimentación incompleta y/o inadecuada de los formularios de notificación. Conclusión: Se ha llegado a la conclusión de que es necesario mejorar las medidas de prevención de la sífilis en los servicios de atención primaria de la salud.

Palabras clave: Sífilis, Epidemiología, Infección de transmisión sexual.

${ }^{1}$ Universidade de Franca (UNIFRAN), Franca - SP. *E-mail: caiormoreno@gmail.com 


\section{INTRODUÇÃO}

As Infecções Sexualmente Transmissíveis (IST) são causadas por vírus, bactérias ou outros microrganismos. A transmissão ocorre, principalmente, através do contato sexual (oral, vaginal, anal) sem o uso de preservativo, com uma pessoa infectada. No entanto, a transmissão de uma doença sexualmente transmissível pode acontecer por outras vias, a exemplo da transmissão vertical, sendo esta da mãe para o feto durante a gestação, ou até mesmo durante o parto ou a amamentação (AZEVEDO AEBI, et al., 2018).

As IST são consideradas como um problema de saúde pública, alcançando âmbito global. Os infectados são mais vulneráveis à associação de mais de uma IST e existe relação com o aumento da mortalidade materna e infantil. Estas infecções possuem altas taxas de incidência e prevalência, apresentando graves complicações (BRASIL, 2015).

A Organização Mundial de Saúde (OMS) estima que ocorrem um milhão de casos de IST por dia. Dentre as diversas IST, sífilis, clamídia, gonorreia e tricomoníase, a patologia causada pelo Treponema pallidum é uma das principais enfermidades em escala mundial e afeta mais de 12 milhões de indivíduos (BRASIL, 2018).

A sífilis apresenta um crescimento exponencial em diversas países, sendo que no Brasil tal quadro está em expansão, tendo apresentado no ano de 2010 cerca de 2 casos para cada 100 mil habitantes e já no ano de 2017, o valor apresentado foi de 58,1 casos para cada 100 mil habitantes. Concomitantemente no ano de 2017, o Sistema Único de Saúde (SUS) destinou 2,8 milhões de dólares em IST, sendo grande parte desta parcela destinada para o tratamento da sífilis, demonstrando que a doença é um relevante problema de saúde no Brasil (BRASIL, 2018).

A sífilis é uma doença sistêmica causada por uma bactéria gram-negativa, do grupo das espiroquetas, denominada $T$. pallidum. A transmissão é predominantemente sexual, sendo mais contagiosa nas fases iniciais da doença. É uma das IST mais relevantes para a saúde em âmbito geral, pois se não tratada precocemente, pode evoluir para uma enfermidade crônica e com sequela irreversível ao longo prazo. Outra via comum de transmissão é a vertical, dando origem à sífilis congênita (FEBRASCO, 2017).

A sífilis congênita é decorrente da disseminação hematogênica do Treponema pallidum da gestante não tratada ou inadequadamente tratada para o seu concepto, por via transplacentária. A transmissão pode ocorrer em qualquer fase da gestação e em qualquer estágio da doença (SÃO PAULO, 2008).

A conservação e a melhoria da saúde materno-infantil são alguns dos objetivos definidos pelo Ministério da Saúde e, para isto, é essencial a atenção pré-natal e puerperal, cuja responsabilidade é do Sistema Único de Saúde. No âmbito da Rede Cegonha, a atenção à mulher durante a gravidez e pós-parto preconiza ações de prevenção e promoção da saúde, além de diagnóstico e tratamento adequado dos problemas que ocorrem neste período. A ocorrência de sífilis congênita é reconhecida como um indicador sensível de avaliação da qualidade da assistência pré-natal (TOMASI E, et al., 2017).

Em relação a sífilis adquirida, transmitida principalmente por via sexual. Tal patologia pode ser classificada em diversos tipos, de acordo com seu quadro clínica e diferenciando-se em relação ao seu tratamento e acompanhamento, sendo estes: primária, secundária e terciaria (KUMAR V, et al., 2010).

A sífilis primária, apresenta um tempo de incubação de 10 a 90 dias, em média de três semanas. A primeira manifestação é caracterizada por uma úlcera rica em treponemas, geralmente única e indolor e elevada, com borda bem definida e regular, além de base endurecida e fundo limpo que ocorre no local de entrada da bactéria (pênis, vulva, vagina, colo uterino, ânus, boca, ou outros locais do tegumento) sendo denominada "cancro duro". A lesão primária é acompanhada de linfadenopatia regional, com duração de 3 a 8 semanas aproximadamente, e seu desaparecimento independente de tratamento. Podendo tal sintomatologia não ser notada ou não ser valorizada pelo paciente (AVELEIRA JCR e BOTTINO G, 2006; KUMAR V, et al., 2010).

A despeito da sífilis secundária ocorre em média entre 6 semanas a 6 meses após a cicatrização do cancro, ainda que manifestações iniciais, recorrentes ou subentrantes do secundarismo possam ocorrer em um período de até dois anos. Deve-se a proliferação das espiroquetas na pele e tecidos mucocutâneos. As manifestações são muito variáveis. Inicialmente apresenta-se uma erupção macular eritematosa pouco visível (roséola), principalmente no tronco e raiz dos membros. Nessa fase são comuns as placas mucosas, lesões 
acinzentadas e pouco visíveis nas mucosas. As lesões cutâneas progridem para lesões mais evidentes papulosas eritematosa acastanhadas que podem atingir todo tegumento sendo frequentes nos genitais (AVELEIRA JCR e BOTTINO G, 2006).

A sífilis terciária, uma das formas mais graves da infecção treponêmica, tal estágio é raro e ocorre aproximadamente em 15 a 25\% das infecções não tratadas, após um período variável de latência, podendo surgir entre um a 40 anos depois do início da infecção. A inflamação causada pela sífilis nesse estágio provoca destruição tecidual. É comum o acometimento do sistema nervoso e do sistema cardiovascular. Além disso, verifica-se a formação de gomas sifilíticas (tumorações com tendência a liquefação) na pele, mucosas, ossos ou qualquer tecido. As lesões podem causar desfiguração, incapacidade e até morte (KUMAR V, et al., 2010).

A sífilis pode resultar em complicações cardiovascular, sendo o acometimento mais comum a aortite, que pode resultar em aneurisma e insuficiência da válvula aórtica. Ademais, uma das complicações mais temida, é o acometimento meníngeo por parte dos treponemas, resultando em neurossífilis. Além disso, a sífilis e o vírus HIV apresentam intensa interação, pois ambos são infecções transmitidas principalmente pela via sexual e aumentam sua importância porque lesões genitais ulceradas aumentam o risco de transmissão do vírus imunodeficiência humana (AVELEIRA JCR e BOTTINO G, 2006; BRASIL, 2016).

A descoberta do T. pallidum é datada do ano de 1905, desde de então ocorreu diversas buscas e pesquisas em relação a patologia sistêmica causada por tal bactéria (SOUZA EM, 2005). Na contemporaneidade, o diagnóstico pode ser realizado por meio de testes rápidos. Esses testes são de fácil execução e sua leitura simples possibilita a investigação da sífilis em locais sem infraestrutura laboratorial, inclusive de difícil acesso. Além disso, por gerar resultados em até 30 minutos, os testes rápidos eliminam o risco de o paciente não voltar ao sistema de saúde para saber seu resultado e, dessa forma, possibilita o seguimento imediato do tratamento do indivíduo. Estes são testes não treponêmicos, a exemplo do VDRL que detecta anticorpos anticardiolipinas, enquanto os testes treponêmicos detectam anticorpos específicos contra componentes celulares dos treponemas (BRASIL, 2010).

O diagnóstico da sífilis é basicamente sorológico, por isso a importância de todas as gestantes serem testadas já na primeira consulta de pré-natal no primeiro trimestre de gestação, devendo repetir a sorologia no início do terceiro trimestre para que, se necessário, a terapêutica adequada seja instituída em tempo oportuno. Em relação ao tratamento, este consiste no emprego da penicilina como primeira escolha e nas doses adequadas para cada momento específico e acometimento da patologia (PADOVANI C, et al., 2018).

No tocante à perspectiva nacional em relação aos casos sífilis no ano de 2019 , foram notificados ao Sistema de Informação de Agravos de Notificação (SINAN) 152.915 casos de sífilis adquirida (taxa de detecção de 72,8 casos/100.000 habitantes); 61.127 casos de sífilis em gestantes (taxa de detecção de 20,8/1.000 nascidos vivos); 24.130 casos de sífilis congênita (taxa de incidência de 8,2/1.000 nascidos vivos); e 173 óbitos por sífilis congênita (taxa de mortalidade de 5,9/100.000 nascidos vivos) (BRASIL, 2020).

Em resumo, a epidemiologia determina a frequência e a distribuição das doenças nas coletividades humanas, apresentando como meta descrever a distribuição e a magnitude dos problemas de saúde da população, além de proporcionar dados essenciais para o planejamento, execução e avaliação das ações de prevenção, controle e tratamento das doenças, bem como para estabelecer prioridades e buscar identificar fatores etiológicos na gênese das enfermidades (CRUZ O, 2008).

Ademais, tal artigo apresenta como meta a descrição do perfil clínico epidemiológico dos pacientes acometidos por sífilis, em um município no interior do estado de São Paulo, a partir dos dados fornecidos pela vigilância epidemiológica municipal, buscando compreender sobre os dados encontrados e suas particularidades.

\section{MÉTODOS}

Trata-se de um estudo observacional descritivo retrospectivo, no qual foi analisado o perfil epidemiológico dos casos de sífilis notificados no município de Franca (SP), a partir dos dados fornecidos pela vigilância epidemiológica municipal, por meio de informações contidas no SINAN. 
Tem como interesse apresentar os dados dos casos de sífilis adquirida, sífilis gestacional e sífilis congênita. Foi escolhido trabalhar com a série histórica a partir do ano de 2009 , um ano antes da notificação da sífilis adquirida, até dados do ano de 2018.

Durante este estudo, foram levantadas as variáveis sociodemográficas (faixa etária, sexo e escolaridade) e os fatores epidemiológicos (idade gestacional do diagnóstico e classificação clínica) de cada paciente, além dos indicadores que se relacionam diretamente à assistência municipal em saúde (momento do diagnóstico da doença materna, óbito pelo agravo, tratamento do parceiro).

Para isso, os dados foram coletados na secretaria municipal de vigilância epidemiológica do município por estudantes de medicina sob supervisão do pesquisador responsável. Tanto o armazenamento quanto as aplicações dos resultados foram realizadas por meio do software Microsoft Excel e Word. A coleta de dados foi efetuada a partir de uma tabela criada pelos próprios estudantes, visando facilitar a compreensão dos dados.

Os critérios de inclusão são todos os pacientes com presença de sífilis, notificados no município de Franca. E os de exclusão, por óbvio, aqueles que não tenham diagnóstico comprovado de sífilis. Destaca-se a limitação do presente estudo ao utilizar fontes de dados secundários, do SINAN, com prováveis sub-registros e/ou subnotificações de casos.

\section{RESULTADOS}

Franca é um município composto por cerca de 350 mil habitantes, onde durante o período de 2009 a 2018 foram notificados um total de 1.088 casos de sífilis, resultando em um acometimento municipal de $0,31 \%$ da população. Diante deste total de casos, 568 eram compostos de sífilis adquirida, 441 de indivíduos com sífilis gestacional e 79 de pessoas com sífilis congênita. O ano que apresentou maior notificação de sífilis foi 2018, sendo 219 casos, enquanto o ano que apresentou menor notificação foi $2011 \mathrm{com}$ apenas 26 casos. Podese verificar estes dados através do Gráfico 1 e os demais tabelas que integram o presente estudo.

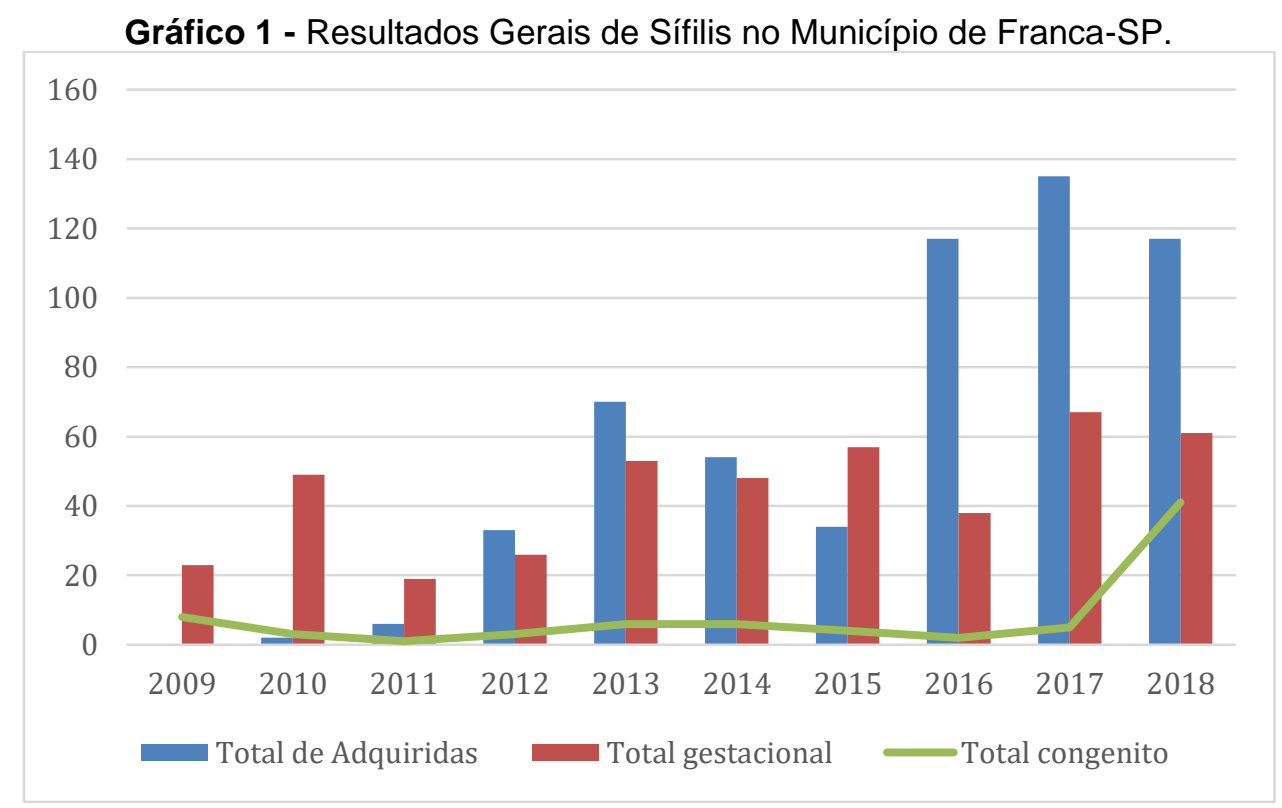

Fonte: Moreno CRP, et al., 2020.

A tabela 1 é composta pela distribuição dos indicadores em sífilis adquirida. Em relação à idade, pode-se analisar um maior número de casos nos indivíduos de 30-39 anos, com 130 casos (22,89\%). Em relação ao sexo pode-se notar um predomínio masculino, com 387 casos $(68,13 \%)$. Sob a perspectiva da raça, ocorre uma preponderância da doença na raça branca com 287 casos (50,53\%). Já em relação à escolaridade, classificação clínica (primária, secundária, terciária ou latente), título, esquema terapêutico e parceiro ou parceira tratado concomitantemente, todos estes quesitos apresentaram o item ignorado em maior proporção. 
Tabela 1 - Resultados obtidos acerca da sífilis adquirida, no município de Franca-SP, no intervalo de 2009-2018.

\begin{tabular}{|c|c|c|}
\hline Variável & $\mathrm{n}$ & $(\%)$ \\
\hline \multicolumn{3}{|l|}{ Idade } \\
\hline $10-14$ & 4 & $(0,70)$ \\
\hline $15-19$ & 75 & $(13,20)$ \\
\hline $20-24$ & 106 & $(18,66)$ \\
\hline $25-29$ & 103 & $(18,13)$ \\
\hline $30-39$ & 130 & $(22,89)$ \\
\hline $40-49$ & 80 & $(14,08)$ \\
\hline $50-59$ & 45 & $(7,92)$ \\
\hline $60-69$ & 18 & $(3,17)$ \\
\hline $70-79$ & 7 & $(1,23)$ \\
\hline \multicolumn{3}{|l|}{ Sexo } \\
\hline Masculino & 387 & $(68,13)$ \\
\hline Feminino & 180 & $(31,69)$ \\
\hline Ignorado & 1 & $(0,18)$ \\
\hline \multicolumn{3}{|l|}{ Raça/Cor } \\
\hline Branca & 287 & $(50,53)$ \\
\hline Preta & 62 & $(10,92)$ \\
\hline Amarela & 1 & $(0,18)$ \\
\hline Parda & 109 & $(19,19)$ \\
\hline Indígena & 0 & $(0,00)$ \\
\hline Ignorado & 108 & $(19,01)$ \\
\hline \multicolumn{3}{|l|}{ Escolaridade } \\
\hline Analfabeto & 0 & $(0,00)$ \\
\hline $1^{\text {a }}$ a $4^{\text {a }}$ série incompleto $\mathrm{EF}$ & 10 & $(1,76)$ \\
\hline $1^{\text {a }}$ a $4^{\text {a }}$ série completo EF & 14 & $(2,46)$ \\
\hline $5^{\mathrm{a}}$ a $8^{\mathrm{a}}$ série incompleto EF & 57 & $(10,04)$ \\
\hline Ensino fundamental completo & 16 & $(2,82)$ \\
\hline Ensino médio incompleto & 42 & $(7,39)$ \\
\hline Ensino médio completo & 85 & $(14,96)$ \\
\hline Educação superior incompleta & 10 & $(1,76)$ \\
\hline Educação superior completa & 20 & $(3,52)$ \\
\hline lgnorado & 311 & $(54,75)$ \\
\hline Não se aplica & 3 & $(0,53)$ \\
\hline \multicolumn{3}{|l|}{ Classificação Clínica } \\
\hline Primária & 53 & $(9,33)$ \\
\hline Secundária & 104 & $(18,31)$ \\
\hline Terciária & 44 & $(7,75)$ \\
\hline Latente & 61 & $(10,74)$ \\
\hline Ignorado & 306 & $(53,87)$ \\
\hline \multicolumn{3}{|l|}{ Teste não treponêmico } \\
\hline Reagente & 454 & $(79,93)$ \\
\hline Não reagente & 4 & $(0,70)$ \\
\hline Não realizado & 1 & $(0,18)$ \\
\hline Ignorado & 109 & $(19,19)$ \\
\hline \multicolumn{3}{|l|}{ Título } \\
\hline Proporção 01:02 & 52 & $(9,15)$ \\
\hline Proporção 01:04 & 47 & $(8,27)$ \\
\hline Proporção 01:08 & 57 & $(10,04)$ \\
\hline Proporção 01:16 & 70 & $(12,32)$ \\
\hline Proporção 01:32 & 68 & $(11,97)$ \\
\hline Proporção 01:64 & 45 & $(7,92)$ \\
\hline Proporção 01:128 & 49 & $(8,63)$ \\
\hline Ignorado & 180 & $(31,69)$ \\
\hline \multicolumn{3}{|l|}{ Teste treponêmico } \\
\hline Reagente & 342 & $(60,21)$ \\
\hline Não reagente & 4 & $(0,70)$ \\
\hline Não realizado & 36 & $(6,34)$ \\
\hline Ignorado & 186 & $(32,75)$ \\
\hline \multicolumn{3}{|l|}{ Esquema terapêutico } \\
\hline PG Benzatina 2.4 & 46 & $(8,10)$ \\
\hline PG Benzatina 4.8 & 145 & $(25,53)$ \\
\hline PG Benzatina 7.2 & 171 & $(30,11)$ \\
\hline Outro esquema & 8 & $(1,41)$ \\
\hline Não realizado & 10 & $(1,76)$ \\
\hline Ignorado & 188 & $(33,10)$ \\
\hline \multicolumn{3}{|c|}{ Parceiro tratado concomitantemente } \\
\hline Sim & 0 & $(0,00)$ \\
\hline Não & 4 & $(0,70)$ \\
\hline Ignorado & 564 & $(99,30)$ \\
\hline Total & 568 & $(100)$ \\
\hline
\end{tabular}

Fonte: Moreno CRP, et al., 2020. 
A Tabela 2 é formada pelo arranjo dos indicadores de sífilis gestacional. Em relação à idade, pode-se verificar um maior número de casos nas grávidas entre os 20-24 anos de idade, com 144 casos (32,65\%), sendo o $1^{\circ}$ trimestre da gestação com maior número de diagnóstico, totalizando 150 gestantes (34,01\%). Em relação à escolaridade e classificação clínica, a maioria foi ignorada. O esquema terapêutico mais empregado na sífilis gestacional foi penicilina benzatina $7.200 .000 \mathrm{UI}$ com 221 mulheres (50,1\%). Sob a óptica do parceiro tratado concomitantemente, 182 mulheres $(41,27 \%)$ relataram que o parceiro não realizou o tratamento simultaneamente.

Tabela 2 - Dados computados acerca da sífilis gestacional, no município de Franca-SP, no intervalo de 2009-2018.

\begin{tabular}{|c|c|c|}
\hline Variável & $\mathbf{n}$ & $(\%)$ \\
\hline \multicolumn{3}{|l|}{ Tdade } \\
\hline $10-14$ & 7 & $(1,59)$ \\
\hline $15-19$ & 100 & $(22,68)$ \\
\hline $20-24$ & 144 & $(32,65)$ \\
\hline $25-29$ & 94 & $(21,32)$ \\
\hline $30-39$ & 91 & $(20,63)$ \\
\hline $40-49$ & 5 & $(1,13)$ \\
\hline \multicolumn{3}{|l|}{ Trimestre } \\
\hline 10 trimestre & 150 & $(34,01)$ \\
\hline $2^{\circ}$ trimestre & 87 & $(19,73)$ \\
\hline $3^{\circ}$ trimestre & 113 & $(25,62)$ \\
\hline Ignorado & 91 & $(20,63)$ \\
\hline Aborto & 0 & $(0,00)$ \\
\hline Não se aplica & 0 & $(0,00)$ \\
\hline \multicolumn{3}{|l|}{ Raça/Cor } \\
\hline Branca & 239 & $(54,20)$ \\
\hline Preta & 39 & $(8,84)$ \\
\hline Amarela & 0 & $(0,00)$ \\
\hline Parda & 59 & $(13,38)$ \\
\hline Indígena & 0 & $(0,00)^{\prime}$ \\
\hline Ignorado & 104 & $(23,58)$ \\
\hline \multicolumn{3}{|l|}{ Escolaridade } \\
\hline Analfabeto & 0 & $(0,00)$ \\
\hline $1^{\mathrm{a}}$ a $4^{\mathrm{a}}$ série incompleto EF & 44 & $(9,98)$ \\
\hline $1^{\text {a }}$ a $4^{\text {a }}$ série completo EF & 10 & $(2,27)$ \\
\hline $5^{\mathrm{a}}$ a $8^{\mathrm{a}}$ série incompleto EF & 38 & $(8,62)$ \\
\hline Ensino fundamental completo & 40 & $(9,07)$ \\
\hline Ensino médio incompleto & 75 & $(17,01)$ \\
\hline Ensino médio completo & 53 & $(12,02)$ \\
\hline Educação superior incompleta & 0 & $(0,00)$ \\
\hline Educação superior completa & 3 & $(0,68)$ \\
\hline Ignorado & 169 & $(38,32)$ \\
\hline Não se aplica & 0 & $(0,00)$ \\
\hline \multicolumn{3}{|l|}{ Classificação Clínica } \\
\hline Primária & 157 & $(35,60)$ \\
\hline Secundária & 21 & $(4,76)$ \\
\hline Terciária & 10 & $(2,27)$ \\
\hline Latente & 116 & $(26,30)$ \\
\hline Ignorado & 137 & $(31,07)$ \\
\hline \multicolumn{3}{|l|}{ Teste não treponêmico } \\
\hline Reagente & 437 & $(99,09)$ \\
\hline Não reagente & 2 & $(0,45)$ \\
\hline Não realizado & 1 & $(0,23)$ \\
\hline Ignorado & 1 & $(0,23)$ \\
\hline \multicolumn{3}{|l|}{ Títuॉo } \\
\hline Proporção 01:02 & 73 & $(16,55)$ \\
\hline Proporção 01:04 & 71 & $(16,10)$ \\
\hline Proporção 01:08 & 66 & $(14,97)$ \\
\hline Proporção 01:16 & 82 & $(18,59)$ \\
\hline Proporção 01:32 & 82 & $(18,59)$ \\
\hline Proporção 01:64 & 41 & $(9,30)^{\prime}$ \\
\hline Proporção 01:128 & 21 & $(4,76)$ \\
\hline Ignorado & 5 & $(1,13)$ \\
\hline \multicolumn{3}{|l|}{ Teste treponêmico } \\
\hline Reagente & 301 & $(68,25)$ \\
\hline Não reagente & 10 & $(2,27)$ \\
\hline Não realizado & 89 & $(20,18)$ \\
\hline Ignorado & 41 & $(9,30)$ \\
\hline \multicolumn{3}{|l|}{ Esquema terapêutico } \\
\hline PG Benzatina 2.4 & 123 & $(27,89)$ \\
\hline PG Benzatina 4.8 & 37 & $(8,39)$ \\
\hline PG Benzatina 7.2 & 221 & $(50,11)$ \\
\hline Outro esquema & 35 & $(7,94)$ \\
\hline Não realizado & 11 & $(2,49)$ \\
\hline lgnorado & 14 & $(3,17)$ \\
\hline \multicolumn{3}{|c|}{ Parceíro tratado concomitantemente } \\
\hline $\operatorname{Sim}$ & 157 & $(35,60)$ \\
\hline Não & 182 & $(41,27)$ \\
\hline Ignorado & 102 & $(23,13)$ \\
\hline Total & 441 & $(100,00)$ \\
\hline
\end{tabular}

Fonte: Moreno CRP, et al., 2020. 
A Tabela 3 representa a distribuição sociodemográfica dos casos de sífilis congênita. Em relação ao sexo e raça do recém-nascido, apresentou maioria ignorada. Em relação à mãe, a maior proporção em relação a idade foi entre $25-29$ anos $(29,11 \%)$, sendo a raça branca mais presente $63,33 \%$ com uma escolaridade de maior proporção de ensino médio completo $(22,78 \%)$. Ademais, verifica-se uma grande proporção de ignorados em todas as variáveis.

Tabela 3 - Apuração dos dados sociodemográficos de pacientes com sífilis congênita, no município de Franca-SP, no intervalo de 2009-2018.

\begin{tabular}{|c|c|c|}
\hline Variável & $\mathbf{n}$ & $(\%)$ \\
\hline \multicolumn{3}{|l|}{ Sexo } \\
\hline Masculino & 26 & $(32,91)$ \\
\hline Feminino & 26 & $(32,91)$ \\
\hline Ignorado & 27 & $(34,18)$ \\
\hline \multicolumn{3}{|l|}{ Raça/Cor } \\
\hline Branca & 34 & $(43,04)$ \\
\hline Preta & 2 & $(2,53)$ \\
\hline Amarela & 0 & $(0,00)$ \\
\hline Parda & 4 & $(5,06)$ \\
\hline Indígena & 0 & $(0,00)$ \\
\hline Ignorado & 39 & $(49,37)$ \\
\hline \multicolumn{3}{|l|}{ Idade da mãe } \\
\hline Ignorado & 5 & $(6,33)$ \\
\hline $10-14$ & 2 & $(2,53)$ \\
\hline $15-19$ & 16 & $(20,25)$ \\
\hline $20-24$ & 18 & $(22,78)$ \\
\hline $25-29$ & 23 & $(29,11)$ \\
\hline $30-39$ & 14 & $(17,72)$ \\
\hline $40-49$ & 1 & $(1,27)$ \\
\hline \multicolumn{3}{|l|}{ Raça/Cor da Mãe } \\
\hline Branca & 50 & $(63,29)$ \\
\hline Preta & 6 & $(7,59)$ \\
\hline Amarela & 0 & $(0,00)$ \\
\hline Parda & 14 & $(17,72)$ \\
\hline Indígena & 0 & $(0,00)$ \\
\hline Ignorado & 9 & $(11,39)$ \\
\hline \multicolumn{3}{|l|}{ Escolaridade da Mãe } \\
\hline Analfabeto & 0 & $(0,00)$ \\
\hline $1^{\mathrm{a}}$ a $4^{\mathrm{a}}$ série incompleto EF & 11 & $(13,92)$ \\
\hline $1^{\mathrm{a}}$ a $4^{\mathrm{a}}$ série completo EF & 3 & $(3,80)$ \\
\hline $5^{\mathrm{a}}$ a $8^{\mathrm{a}}$ série incompleto EF & 10 & $(12,66)$ \\
\hline Ensino fundamental completo & 8 & $(10,13)$ \\
\hline Ensino médio incompleto & 13 & $(16,46)$ \\
\hline Ensino médio completo & 18 & $(22,78)$ \\
\hline Educação superior incompleta & 0 & $(0,00)$ \\
\hline Educação superior completa & 0 & $(0,00)$ \\
\hline Ignorado & 16 & $(20,25)$ \\
\hline Não se aplica & 0 & $(0,00)$ \\
\hline Total & 79 & $(100)$ \\
\hline
\end{tabular}

Fonte: Moreno CRP, et al., 2020.

A Tabela 4 representa a distribuição dos indicadores de sífilis congênita. $O$ pré-natal foi realizado na maioria dos casos, 64 mulheres $(81,01 \%)$ e o diagnóstico na mãe foi verificado no pré-natal em 50 casos $(63,29 \%)$. No entanto, o tratamento da mãe apresentou uma divisão equitativa entre tratamento adequado ou não realizado de 31 casos $(39,24 \%)$ para ambos. O teste não treponêmico do liquor resultou em não reagente em 50 indivíduos $(63,29 \%)$. O esquema mais empregado na sífilis congênita foi penicilina cristalina 100.000 a 150.00010 dias em 27 seres (34,18\%). E a evolução dos casos, com na grande maioria vivo 69 indivíduos $(87,34 \%)$. 
Tabela 4 - Relatos dos números de sífilis congênita, no município de Franca-SP, no intervalo de 2009-2018.

\begin{tabular}{|c|c|c|}
\hline Variável & $\mathrm{n}$ & $(\%)$ \\
\hline \multicolumn{3}{|c|}{ Realizou pré natal na gestação } \\
\hline Sim & 64 & $(81,01)$ \\
\hline Não & 12 & $(15,19)$ \\
\hline Ignorado & 3 & $(3,80)$ \\
\hline \multicolumn{3}{|c|}{ Diagnóstico de Sífilis na mãe } \\
\hline Pré-natal & 50 & $(63,29)$ \\
\hline No momento do parto & 19 & $(24,05)$ \\
\hline Após o parto & 2 & $(2,53)$ \\
\hline Não realizado & 0 & $(0,00)$ \\
\hline Ignorado & 8 & $(10,13)$ \\
\hline \multicolumn{3}{|c|}{ Teste não treponêmico da mãe no parto } \\
\hline Reagente & 71 & $(89,87)$ \\
\hline Não reagente & 5 & $(6,33)$ \\
\hline Não realizado & 1 & $(1,27)$ \\
\hline Ignorado & 2 & $(2,53)$ \\
\hline \multicolumn{3}{|l|}{ Título da mãe no parto } \\
\hline Proporção 01:01 & 3 & $(3,80)$ \\
\hline Proporção 01:02 & 16 & $(20,25)$ \\
\hline Proporção 01:04 & 14 & $(17,72)$ \\
\hline Proporção 01:08 & 4 & $(5,06)$ \\
\hline Proporção 01:16 & 9 & $(11,39)$ \\
\hline Proporção 01:32 & 11 & $(13,92)$ \\
\hline Proporção 01:64 & 6 & $(7,59)$ \\
\hline Proporção 01:128 & 4 & $(5,06)$ \\
\hline Ignorado & 3 & $(3,80)$ \\
\hline Não reagente & 9 & $(11,39)$ \\
\hline \multicolumn{3}{|l|}{ Teste treponêmico parto } \\
\hline Reagente & 4 & $(5,06)$ \\
\hline Não reagente & 2 & $(2,53)$ \\
\hline Não realizado & 60 & $(75,95)$ \\
\hline Ignorado & 13 & $(16,46)$ \\
\hline \multicolumn{3}{|l|}{ Tratamento da Mãe } \\
\hline Adequado & 31 & $(39,24)$ \\
\hline Inadequada & 31 & $(39,24)$ \\
\hline Não realizado & 5 & $(6,33)$ \\
\hline Ignorado & 12 & $(15,19)$ \\
\hline \multicolumn{3}{|c|}{ Teste não treponêmico do RN } \\
\hline Reagente & 66 & $(83,54)$ \\
\hline Não reagente & 6 & $(7,59)$ \\
\hline Não realizado & 1 & $(1,27)$ \\
\hline Ignorado & 6 & $(7,59)$ \\
\hline \multicolumn{3}{|l|}{ Título do RN } \\
\hline Proporção 01:01 & 4 & $(5,06)$ \\
\hline Proporção 01:02 & 16 & $(20,25)$ \\
\hline Proporção 01:04 & 5 & $(6,33)$ \\
\hline Proporção 01:08 & 12 & $(15,19)$ \\
\hline Proporção 01:16 & 9 & $(11,39)$ \\
\hline Proporção 01:32 & 5 & $(6,33)$ \\
\hline Proporção 01:64 & 4 & $(5,06)$ \\
\hline Proporção 01:128 & 1 & $(1,27)$ \\
\hline Ignorado & 4 & $(5,06)$ \\
\hline Não reagente & 19 & $(24,05)$ \\
\hline \multicolumn{3}{|c|}{ Teste não treponêmico do liquor } \\
\hline Reagente & 2 & $(2,53)$ \\
\hline Não reagente & 50 & $(63,29)$ \\
\hline Não realizado & 16 & $(20,25)$ \\
\hline Ignorado & 11 & $(13,92)$ \\
\hline
\end{tabular}




\begin{tabular}{|c|c|c|}
\hline Variável & $\mathrm{n}$ & $(\%)$ \\
\hline \multicolumn{3}{|l|}{ Título no liquor } \\
\hline Proporção 01:01 & 27 & $(34,18)$ \\
\hline Proporção 01:02 & 1 & $(1,27)$ \\
\hline Proporção 01:04 & 1 & $(1,27)$ \\
\hline Proporção 01:08 & 0 & $(0,00)$ \\
\hline Proporção 01:16 & 0 & $(0,00)$ \\
\hline Proporção 01:32 & 0 & $(0,00)$ \\
\hline Proporção 01:64 & 0 & $(0,00)$ \\
\hline Proporção 01:128 & 0 & $(0,00)$ \\
\hline Ignorado & 5 & $(6,33)$ \\
\hline Não reagente & 45 & $(56,96)$ \\
\hline \multicolumn{3}{|l|}{ Evidencia de treponêma pallidum } \\
\hline Sim & 0 & $(0,00)$ \\
\hline Não & 6 & $(7,59)$ \\
\hline Não Realizado & 36 & $(45,57)$ \\
\hline Ignorado & 37 & $(46,84)$ \\
\hline Diagnóstico clínico & 0 & $(0,00)$ \\
\hline Assintomático & 57 & $(72,15)$ \\
\hline Sintomático & 12 & $(15,19)$ \\
\hline Não se aplica & 1 & $(1,27)$ \\
\hline Ignorado & 9 & $(11,39)$ \\
\hline \multicolumn{3}{|l|}{ Esquema de tratamento } \\
\hline PG Cristalina 100.000 a 150.00010 dias & 27 & $(34,18)$ \\
\hline PG procaína 50.00010 dias & 22 & $(27,85)$ \\
\hline PG Benzatina 50.000 & 8 & $(10,13)$ \\
\hline Outro esquema & 10 & $(12,66)$ \\
\hline Não realizado & 1 & $(1,27)$ \\
\hline Ignorado & 11 & $(13,92)$ \\
\hline \multicolumn{3}{|l|}{ Evolução do caso } \\
\hline Vivo & 69 & $(87,34)$ \\
\hline Óbito por sífilis congênita & 0 & $(0,00)$ \\
\hline Óbito por outras causas & 1 & $(1,27)$ \\
\hline Aborto & 0 & $(0,00)$ \\
\hline Natimorto & 1 & $(1,27)$ \\
\hline Ignorado & 8 & $(10,13)$ \\
\hline Total & 79 & $(100,00)$ \\
\hline
\end{tabular}

Fonte: Moreno CRP, et al., 2020.

\section{DISCUSSÃO}

Em relação aos dados nacionais, pode-se observar que no ano de 2019 , foram notificados ao SINAN 152.915 casos de sífilis adquirida (taxa de detecção de 72,8 casos/100.000 habitantes); 61.127 casos de sífilis em gestantes (taxa de detecção de 20,8/1.000 nascidos vivos); 24.130 casos de sífilis congênita (taxa de incidência de 8,2/1.000 nascidos vivos) (BRASIL, 2020).

O SINAN é um sistema alimentado, pela notificação e investigação de casos de doenças e agravos que constam na lista nacional de doenças de notificação compulsória (Portaria de Consolidação no 4 , de 28 de Setembro de 2017). Dessa maneira, o Ministério da Saúde monitora a infecção por meio das notificações de sífilis adquirida, que abrange toda a população, e compreendem todos os casos de sífilis (adquirida, congênita, em gestante), com a finalidade de realizar um diagnóstico precoce e tratar tal patologia, além de evitar a transmissão vertical e complicações para mulheres e crianças. O tratamento é realizado com a aplicação de penicilina benzatina, oferecida gratuitamente no SUS (BRASIL, 2020).

A notificação compulsória de sífilis congênita em todo o território nacional foi instituída por meio da Portaria no 542, de 22 de dezembro de 1986; a notificação compulsória de sífilis em gestante foi instituída pela Portaria no 33, 14 de julho de 2005; e, por último, notificação compulsória de sífilis adquirida, por intermédio da Portaria № 2.472, de 31 de agosto de 2010 (BRASIL, 2016). 
Assim como no Brasil, o município de Franca (SP) mantém a proporção em relação aos tipos de sífilis, sendo a adquirida mais presente no munícipio, seguida respectivamente pela gestacional e pela congênita. Em relação à sífilis adquirida, no ano de 2009 nenhum caso foi notificado e tal fato pode ser explicado em razão da notificação compulsória não ser obrigatória à época, vez que teve início apenas quando editadas a Portaria ํㅡㄴ.472, de 31 de agosto de 2010.

Pode-se analisar um aumento do número de casos do ano de 2009 a 2018, assim como no território nacional. Tal aumento do número de casos notificados ao longo da série histórica estudada pode ser atribuído a diversos fatores, como o aumento das notificações, bem como ações de vigilância epidemiológica direcionadas para uma melhor abordagem e identificação dos casos da doença no município (LIMA VC, et al., 2017). Nesse contexto, a política de saúde denominada "Rede Cegonha", instituída em 2011, contribuiu para a ampliação do acesso ao diagnóstico de sífilis em gestante no país (BRASIL, 2016).

Em relação à idade, a maioria dos indivíduos suscetíveis a patologia estava entre os 20 e 39 anos, sendo está uma faixa sexualmente ativa. No entanto, nos extremos etários também pode-se verificar a ocorrência de certos casos, a exemplo dos idosos, onde pode servir para uma maior orientação em relação ao uso de preservativos nesta faixa etária.

Sob a perspectiva de cor/raça, este pode ser um parâmetro relevante em diversas patologias, entretanto apresenta uma dificuldade, pois este é um critério autodeclarado podendo resultar em resultado falso-positivo. No entanto, as pacientes declaram-se brancas ou tal indicador foi ignorado.

No que se refere a escolaridade, parâmetro de grande valia na busca da compreensão epidemiológica da lues no munícipio, independentemente do tipo de sífilis encontrou-se a maioria dos casos marcados como ignorado. A escolaridade materna, neste estudo evidenciou resultados diferentes dos encontrados em estudos realizados na Bahia, Minas Gerais e território nordestino cuja maior predominância foi entre indivíduos com ensino fundamental incompleto (LIMA VC, et al., 2017; LIMA MR, et al., 2013; OLIVEIRA JS e SANTOS JV, 2015). No entanto, assemelha-se no elevado número de fichas com o campo da escolaridade em branco ou preenchido como ignorado, o que compromete uma análise fidedigna dessa informação.

Alguns parâmetros como ocupação (até por isso retirado dos parâmetros considerados neste trabalho) foram ignorados na maioria dos resultados, independentemente do tipo de sífilis. O preenchimento inadequado pode resultar em dados epidemiológico equivocados, resultando em problemáticas no âmbito de saúde. A não realização correta da notificação compulsória pode estar relacionada especialmente com a sobrecarga de trabalho dos profissionais de saúde e com a incompreensão acerca da importância dessa notificação no contexto da atenção (ALVARES JK, 2015).

Em relação à sífilis adquirida, a maior predominância ocorreu no sexo masculino. Tal achado pode apresentar correlação com os sinais e sintomas da sífilis, pois a sífilis primária apresenta como apresentação cancro indolor. No entanto, nas mulheres, tais lesões podem situar-se dentro do canal vaginal, sendo imperceptível a visualização enquanto no sexo masculino é possível observar o cancro, resultando, na procura médica devido a presença de lesões na região genial (AVELEIRA JCR, BOTTINO G, 2006). Ademais, os valores da cultura masculina envolvem maior comportamento de risco à saúde, como não utilização de preservativos, enquanto as mulheres apresentam uma maior procura às consultas de saúde (LEVORATO $C D, 2014)$.

Sob a perspectiva da sífilis gestacional, apenas 11 casos (2,49\%) não apresentaram esquema terapêutico. No entanto, um total de 182 casos $(41,27 \%)$ não apresentaram o tratamento concomitante do parceiro, assim como uma grande parcela das mulheres com sífilis gestacional não realizaram um tratamento adequado, estando suscetíveis à reinfecção e falhas na adequabilidade do tratamento (BRASIL, 2016). Tal nuance, pode resultar em complicações fetais, pois os fetos de gestante com sífilis não tratada serão infectados em 70 a $100 \%$ dos casos (LINS CDM, 2014).

Em relação ao pré-natal, as gestantes demonstraram uma boa adesão $(81,01 \%)$. A assistência no prénatal é um momento de grande valia para a mulher, uma vez que auxilia nas finalidades de diagnóstico, tratamento, promoção da saúde e prevenção de possíveis intercorrências oriundas do período gestacional, 
inclusive previne problemas relacionados à saúde do concepto (BRASIL, 2012). Assim, sendo diagnóstico $63,29 \%$ dos casos de sífilis materna durante o pré-natal, sendo apenas $24,05 \%$ os diagnósticos estabelecidos no momento do parto.

Diante da sífilis congênita, pode-se verificar um tratamento inadequado por parte das mães apresentando uma portagem de $39,24 \%$, enquanto equitativamente $39,24 \%$ realizam o tratamento de forma correta. Tais números servem de alerto, pois, a sífilis congênita pode resultar em diversas complicações como prematuridade, baixo peso ao nascer, hepatomegalia, osteocondrite, aborto e até óbito fetal (BRASIL, 2006). Dessa forma, grande parte das crianças ficaram expostas a estas diversas complicações da sífilis congênita. Por fim, realizou-se teste não treponêmico no liquor para verificação de neurossífilis e $63,29 \%$ do conceptos foram não reagentes. A evolução dos casos resultou em feto vivo em $87,34 \%$ dos casos, enquanto apenas $1,27 \%$ natimorto.

\section{CONCLUSÃO}

A sífilis é uma infecção sexualmente transmissível que pode ser controlada por meio de ações e medidas eficazes de saúde pública, em virtude de apresentar testes diagnósticos sensíveis e tratamento efetivo e de baixo custo. Desta maneira, entendemos que se faz necessário melhorar as ações para prevenção da sífilis nos serviços de saúde da atenção básica, focalizando na detecção precoce da gestante, no diagnóstico e tratamento oportuno da sífilis adquirida, assim como na captação dos parceiros. Além disso, verifica-se um destaque maior dos casos na faixa de adultos jovens, evidenciando que as ações educativas devem ter um enfoque maior para esse grupo de indivíduos. Por fim, os profissionais da saúde devem contribuir com uma maior preocupação em relação a notificação dos casos de sífilis e preenchimento completo das fichas, contribuindo para uma melhor abordagem epidemiológica desta infecção sexualmente transmissível.

\section{REFERÊNCIAS}

1. ALVARES JK, et al. Avaliação da completitude das notificações compulsórias relacionadas ao trabalho registradas por município polo industrial no Brasil, 2007 - 2011. Revista Brasileira de Epidemiologia, 2015.

2. AVELLEIRA JCR, BOTTINO G. Sífilis: diagnóstico, tratamento e controle. Anais brasileiro de dermatologia, 2006.

3. AZEVEDO AEBI, et al. Infecções Sexualmente Transmissíveis na Adolescência. Sociedade Brasileira de Pediatria, 2018,6 (3).

4. BRASIL. Manual do Ministérios de Saúde. Diretrizes para controle da sífilis congênita. Brasília: Secretaria de Vigilância em Saúde, 2006.

5. BRASIL. Manual do Ministérios de Saúde. Sífilis: Estratégias para diagnóstico no Brasil. Brasília, 2010.

6. BRASIL. Manual do Ministérios de Saúde. Caderno de atenção básica: atenção ao pré natal de baixo risco. Brasília: Secretaria de Atenção à Saúde, 2012.

7. BRASIL. Manual do Ministérios de Saúde. Protocolo clínico e Diretrizes Terapêuticas para Atenção Integral às Pessoas com Infecção Sexual Transmissível. Brasília: Ministério da Saúde, 2015.

8. BRASIL. Manual do Ministérios de Saúde. Boletim epidemiológico. Brasília: Secretaria de Vigilância em Saúde, 2016.

9. BRASIL. Manual do Ministérios de Saúde. Manual técnico para diagnóstico da sífilis. Brasília: Secretaria de Vigilância em Saúde, 2016.

10. BRASIL. Manual do Ministérios de Saúde. Boletim epidemiológico. Brasilía: Ministério da Saúde, 49, 2018.

11. BRASIL. Manual do Ministérios de Saúde. AIDS. Doenças de condições crônicas e infecções sexualmente transmissíveis, 2020. Disponivel em: http://www.aids.gov.br/pt-br/noticias/brasil-avanca-no-enfrentamento-sifilis. Acesso em: 11 Novembro 2020.

12. BRASIL. Manual do Ministérios de Saúde. Boletim epidemiológico. Brasília: Secretaria de Vigilância em Saúde, 2020.

13. CRUZ FO. Noções básicas da epidemiologia. In: MONTILLA, D. E. R. Envelhecimento e saúde da pessoa idosa. Rio de Janeiro: Fundação Oswaldo Cruz, 2008.

14. FEBRASCO. FEBRASGO. FEBRASGO, 2017. Disponivel em: https://www.febrasgo.org.br/pt/noticias/item/188-sifilis. Acesso em: 11 Novembro 2020.

15. KUMAR, V, et al. Robbins\&Cotran Patologia: bases patológicas das doenças. 8a. ed. Rio de Janeiro: Elsevier, 2010, 377-381 p.

16. LEVORATO CD, et al. Fatores associados à procura por serviços de saúde numa perspectiva relacionada ao gênero. Ciência \& Saúde Coletiva, 2014, 1263-1274 p.

17. LIMA MG, et al. Incidência e fatores de risco para sífilis congênita em Belo Horizonte, Minas Gerais, 2001-2008. Ciência \& Saúde Coletiva, Rio de Janeiro, 18 (2), 2013.

18. LIMA VC, et al. Perfil epidemiológico dos casos de sífilis congênita em um município de médio porte no nordeste brasileiro. Journal of Health Biological Sciences, 5, 2017, 56-61 p.

19. LINS CDDM. Epidemiologia da sífilis gestacional e congênita no extremo setentrional da Amazônia, Boa Vista, 2014.

20. OLIVEIRA JS, SANTOS JV. Perfil epidemiológico da sífilis congênita no estado da Bahia, do período de 2010 a 2013 . Revista Eletrônica Atualiza Saúde, Salvador, 2 (2), 2015.

21. PADOVANI C, et al. Sífilis na gestação: associação das características maternas e perinatais em região do sul do Brasil. Revista Latino-Americana de Enfermagem, 2018.

22. SOUZA EMD. Há 100 anos, a descoberta do Treponema pallidum. Anais Brasileiro de Dermatologia, 80, 2005.

23. TOMASI E, et al. Qualidade da atenção pré-natal nas rede básica de saúde do Brasil: indicadores e desigualdades sociais. Caderno de Saúde Pública, Pelotas/RS, 33, 2017.

24. SÃO PAULO. Secretaria de Estado da Saúde (SES-SP). Serviço de Vigilância Epidemiológica. Coordenação do Programa Estadual DST/Aids-SP. Coordenadoria de Controle de Doenças (CCD). Sífilis congênita e sífilis na gestação. Revista Saúde Pública, 2008; 42: 768-772. 